\title{
Advances in UV Ground- and Space-based Measurements and Modeling II
}

\author{
James Slusser \\ Wei Gao \\ Colorado State University \\ Natural Resource Ecology Laboratory \\ UV-B Monitoring and Research \\ Program \\ Fort Collins, Colorado 80523 \\ E-mail: sluss@uvb.nrel.colostate.edu \\ Jay R. Herman \\ NASA Goddard Space Flight Center \\ Code 916 \\ Greenbelt, Maryland 20771
}

\section{Introduction}

On August 4-6, 2003, SPIE hosted a conference on UV Ground- and Space-based Measurements, Models, and Effects III in San Diego that attracted 44 papers. We asked the authors of 12 of those papers to revise and enlarge their papers from Proceedings of SPIE Volume 5156 to be included in this special section of the journal Optical Engineering.

\section{What Modulates UV Transmission}

The first two papers are comprehensive surveys. The paper by Kerr provides a summary of the factors affecting UV irradiance at the Earth's surface and includes a comparison of the advantages and disadvantages of various UV instrumentation. Stamnes brings us to the state of the art in atmospheric correction in the presence of aerosols for retrievals of the earth and ocean properties from space. One of the big unknowns is aerosol absorption or single scattering albedo (SSA). Two papers by Krotkov et al. derive SSA from combined measurements of UV shadowband radiometer, direct-Sun photometers, and radiative transfer calculations. Wetzel and Slusser elucidate a new technique to infer surface UV from satellite measurements distinguishing between cloud and snow, and validate their model with surface measurements.

\section{Instrumentation}

Kaifel et al. describe a new highly sensitive 5-channel UV filter radiometer capable of producing the entire spectrum via the neural network method. In a companion paper
Feister et al. provide an intercomparison of this device with a standard spectrometer. A 5-year record of erythemal UV doses measured at two locations by the U.S. Environmental Protection Agency (EPA) Brewer and the U.S. Department of Agriculture (USDA) UV-B broadband meters are compared in the first paper by Kimlin et al. In a second paper, Kimlin et al. investigate techniques for monitoring and maintaining the performance of the Brewer spectrophotometer.

\section{Algorithms}

Dahlback et al. refine their ozone and dose retrievals from a filter radiometer. Bernhard et al. monitor column ozone in real time with a 5-channel filter radiometer. Englessen and Kylling describe a fast 2-stream model that simulates the changing UV irradiance that can be used as a diagnostic to determine the quality of measured spectra.

\section{Conclusion}

These papers represent some of the most recent advances in UV research. New instrumentation and calibration techniques, careful analysis of long-term data, and comparison between data and models are resulting in better understanding of the distribution of UV reaching the Earth. The editors wish to thank the reviewers for the generous gift of their time and experience, and the authors for their original ideas. Thanks go out to the entire staff of SPIE, especially Jonica Todd and Karolyn Labes for their encouragement and outstanding logistical support. 


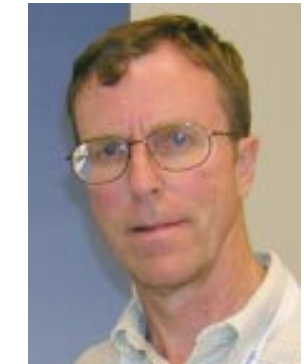

James Slusser studied physics at Western Michigan University and received his bachelor's degree in 1977 and his master's degree in 1980. The next nine years were spent in Chicago, where he was the undergraduate physics laboratory director at Loyola University and later worked as a design engineer for Atlas Electric Devices, serving as a project manager for an ultraviolet outdoor radiometer and temperature probe for weathering chambers. In 1989 he returned to school to study atmospheric science and radiative transfer at the University of Alaska at Fairbanks. His dissertation involved measuring stratospheric nitrogen dioxide and exploring its relationship to ozone abundances. After earning his PhD in 1994, he spent three months at Lauder, New Zealand, doing trace gases analysis using the zenith sky spectroscopy. He then took up a position as a research associate at the Center for Atmospheric Science at Cambridge University in England where he investigated the effects of volcanic aerosols on nitrogen dioxide and ozone abundances in Antarctica. In 1996 he became a research scientist at the USDA UV-B Monitoring and Research Program at Colorado State University. In 1999 he became the director of the program. His current research interests include improving the calibration accuracy and precision of UV measurements and retrievals of trace gas column abundances and aerosol properties. He has authored or co-authored more than 25 papers. He is a member of the AGU, AMS, and SPIE and served as an associate editor of the Journal of Geophysical Research.

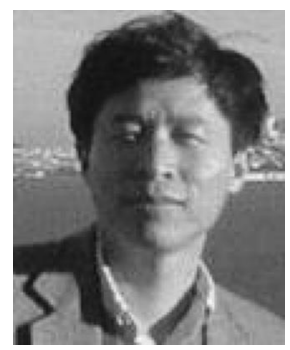

Wei Gao received a BS in applied meteorology from Nanjing Institute of Meteorology, China, and an MS in agromicrometeorology from Mississippi State University. He received his $\mathrm{PhD}$ in applied meteorology from Purdue University. He developed a geometric UV radiation transfer model and estimated the UV-B radiation loading on potentially UV-B sensitive surfaces in vegetation canopies. He did his postdoctoral training at the National Center for Atmospheric Research, Boulder, Colo- rado, where he conducted research on climate change scenario formation, climate change impacts on the biosphere, and analysis of climate variability and extreme climate events. He is currently a research scientist at the USDA UV-B Monitoring and Research Program at Colorado State University. His research interests include atmospheric/vegetation canopy radiation transfer modeling, UV radiation, UV radiation and other climate stress factors influences on plants, total ozone interactions with other atmospheric parameters, impact of climate change, and ecosystem modeling.

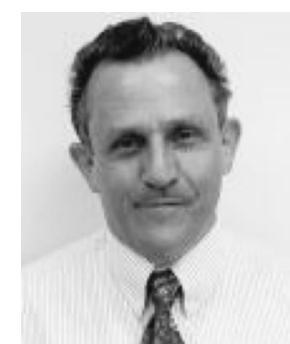

Jay R. Herman received a BS in physics, with a minor in mathematics, from Clarkson College, Potsdam, New York, in 1959, and an MS (1963) and PhD (1965) in physics from Pennsylvania State University. He joined NASA Goddard Space Flight Center, Atmospheric Chemistry and Dynamics Branch, in 1966 where he has specialized in ion chemistry, earth ionosphere, planetary ionospheres, radio wave propagation in plasmas, stratospheric chemistry and modeling, radiative transfer, atmospheric spectroscopy, UV solar flux measurements, ozone inversion algorithms, long-term ozone trend analysis, volcanic aerosols, and physical oceanography. He is currently project scientist for the Deep Space Climate Observatory (DSCOVR) Mission and principal investigator for UV and aerosol projects, the L-2 Interferometer, and the Ocean Radiation Project. 